\title{
The effect of the ragweed sublingual immunotherapy tablet MK-3641 on rescue medication use
}

\author{
Sandra Gawchik', Peter Creticos ${ }^{2}$, Kevin R. Murphy ${ }^{3}$, Gary Berman ${ }^{4}$, David I. Bernstein ${ }^{5}$, Jennifer Maloney ${ }^{6}$, \\ Amarjot Kaur ${ }^{6}$, Hendrik Nolte ${ }^{6^{*}}$ \\ From Canadian Society of Allergy and Clinical Immunology Annual Scientific Meeting 2014 \\ Ottawa, ON, Canada. 23-26 October 2014
}

\section{Background}

Allergic rhinitis with/without conjunctivitis (AR/C) sufferers often rely on pharmacotherapy to relieve symptoms. Although the main goal of immunotherapy is long-term disease modification, reducing or eliminating the need for pharmacotherapy is also an important and desirable treatment goal.

\section{Methods}

Data were pooled from two trials that evaluated the efficacy and safety of short-ragweed sublingual immunotherapy tablet (SLIT-T), MK-3641 (Ambrosia artemisiifolia; Merck/ALK-Abelló). Subjects with ragweed-polleninduced AR/C were randomized $\sim 16$ weeks before the 2010 pollen season to once-daily MK-3641 (6 or $12 \mathrm{Amb}$ a 1-U doses; one trial also included a no-effect dose of 1.5 Amb a 1-U) or placebo. During the trial, all subjects, whether taking MK-3641 or placebo, could use AR/C rescue medication, including oral/ocular antihistamines and intranasal/oral corticosteroids. We examined rescue medication use in all groups.

\section{Results}

In pooled results from the two studies, 159 of 318 (50.0\%) subjects receiving MK-3641 $12 \mathrm{Amb}$ a 1-U and 144 of 324 (44.4\%) subjects receiving $6 \mathrm{Amb}$ a $1-\mathrm{U}$ used no rescue medication over the entire ragweed season, compared with 109 of 340 (32.1\%) subjects receiving placebo. These differences represented $56 \%$ and $38 \%$ improvements over placebo. Similarly, during the peak ragweed season 173 of 311 (55.6\%) subjects and 161 of 317 (50.8\%) subjects in the $12 \mathrm{Amb}$ a 1-U and $6 \mathrm{Amb} 1-\mathrm{U}$ groups, respectively, reported no rescue medication use, in contrast to 136 of

${ }^{6}$ Merck \& Co., Inc., Whitehouse Station, NJ, USA

Full list of author information is available at the end of the article
333 (40.8\%) subjects receiving placebo. Fewer subjects taking 12 and $6 \mathrm{Amb}$ a 1-U (28\% and 19\%, respectively) used oral antihistamine than those taking placebo; $35 \%$ and $28 \%$ fewer subjects used ocular antihistamine; and $43 \%$ and $27 \%$ fewer subjects used intranasal corticosteroid (oral corticosteroid was used by $<5$ subjects in any group, so rates were not calculated).

\section{Conclusions}

Compared with placebo, the SLIT-T treatment MK-3641 reduced rescue-medication use among subjects with ragweed-pollen-induced AR/C.

\section{Trial registration}

ClinicalTrials.gov Identifiers: NCT00783198; NCT00 770315.

\section{Acknowledgements}

Medical writing and editorial assistance was provided by Erin P. Scott, PhD. This assistance was funded by Merck \& Co., Inc., Whitehouse Station, NJ, USA. Editorial assistance was also provided by Jorge Moreno-Cantu, PhD, Global Scientific and Medical Publications, Office of the Chief Medical Officer, Merck \& Co., Inc., Whitehouse Station, NJ, USA.

\section{Authors' details}

${ }^{1}$ Asthma \& Allergy Associates, Chester, PA, USA. ²Department of Medicine, Johns Hopkins University School of Medicine, Baltimore, MD, USA. ${ }^{3}$ Boys Town National Research Hospital, Boys Town, NE, USA. ${ }^{4}$ Minneapolis Allergy \& Asthma Specialists, Minneapolis, MN, USA. ${ }^{5}$ Bernstein Allergy Group, Cincinnati, OH, USA. ${ }^{6}$ Merck \& Co., Inc., Whitehouse Station, NJ, USA.

Published: 18 December 2014

doi:10.1186/1710-1492-10-S2-A32

Cite this article as: Gawchik et al:: The effect of the ragweed sublingual immunotherapy tablet MK-3641 on rescue medication use. Allergy, Asthma and Clinical Immunology 2014 10(Suppl 2):A32. 